\section{WNT signaling, in synergy with T/TBX6, controls Notch signaling by regulating $D 111$ expression in the presomitic mesoderm of mouse embryos}

\author{
Michael Hofmann, ${ }^{1}$ Karin Schuster-Gossler, ${ }^{2}$ \\ Masami Watabe-Rudolph, ${ }^{2}$ Alexander Aulehla, ${ }^{1}$ \\ Bernhard G. Herrmann, ${ }^{1,3,4}$ and Achim Gossler ${ }^{2,5}$ \\ ${ }^{1}$ Max-Planck-Institute of Immunobiology, Stübeweg 51, \\ D-79108 Freiburg, Germany; ${ }^{2}$ Institute for Molecular Biology \\ OE5250, Medizinische Hochschule Hannover, \\ D-30625 Hannover, Germany
}

Notch signaling in the presomitic mesoderm (psm) is critical for somite formation and patterning. Here, we show that WNT signals regulate transcription of the Notch ligand Dll1 in the tailbud and psm. LEF/TCF factors cooperate with TBX6 to activate transcription from the Dll1 promoter in vitro. Mutating either T or LEF/ TCF sites in the Dll1 promoter abolishes reporter gene expression in vitro as well as in the tail bud and psm of transgenic embryos. Our results indicate that WNT activity, in synergy with TBX6, regulates Dll1 transcription and thereby controls Notch activity, somite formation, and patterning.

Received August 11, 2004; revised version accepted September 13, 2004.

The subdivision of the paraxial mesoderm into somites, a metameric series of homologous subunits, is the most obvious sign of segmentation in early vertebrate embryos. During mouse embryogenesis, the first somites form in the posterior headfold region of the embryo around embryonic day 7.75 (E7.75). Subsequently, new somites condense at regular intervals in a strict anteriorto-posterior sequence from the unsegmented so-called presomitic mesoderm (psm) that lies caudally to the first somites. Somite condensation progresses while concomitantly new paraxial mesoderm cells are being generated caudally from the primitive streak and later from the tail bud elongating the embryo posteriorly.

Somite formation is coupled to a molecular oscillator referred to as the segmentation clock, which has been revealed by the cyclic expression of genes in the psm. Expression of cyclic genes is periodic such that one wave of expression passes through the psm during the formation of one somite (Palmeirim et al. 1997; Forsberg et al.

[Keywords: WNT signaling; Notch signaling; D111; somitogenesis; regulation]

${ }^{3}$ Present address: Max-Planck-Institute for Molecular Genetics, Ihnestraße 73, D-14195 Berlin, Germany.

Corresponding authors.

${ }^{4}$ E-MAIL herrmann@molgen.mpg.de; FAX 49-30-8413-1229.

${ }^{5}$ E-MAIL gossler.achim@mh-hannover.de; FAX 49-511-532-4283.

Article and publication are at http://www.genesdev.org/cgi/doi/10.1101/ gad.1248604
1998; Jiang et al. 2000; Jouve et al. 2000; Aulehla et al. 2003). The segmentation clock is closely linked to Notch and WNT signaling activity. Most genes displaying cyclic activity encode components of the Notch pathway (Palmeirim et al. 1997; Forsberg et al. 1998; Jiang et al. 2000; Jouve et al. 2000); one other cyclic gene encodes the WNT pathway component Axin2 (Aulehla et al. 2003). Altered Notch signaling disrupts somite formation and patterning in Xenopus, zebrafish, and mouse embryos (Conlon et al. 1995; Hrabe de Angelis et al. 1997; Jen et al. 1999; Holley et al. 2000; Jiang et al. 2000; Sawada et al. 2000). Furthermore, mutations in some Notch pathway components, which lead to defects in somitogenesis, also affect the expression of cyclic genes (del Barco Barrantes et al. 1999; Jiang et al. 2000; Jouve et al. 2000; Dunwoodie et al. 2002), indicating that Notch signaling is essential for generating cyclic gene expression. Cyclic Lfng gene expression was shown in chick and mouse embryos to be essential for Lfng function (Dale et al. 2003; Serth et al. 2003). Disruption of WNT/ $\beta$-catenin signaling also affects somitogenesis and cyclic expression of Notch pathway components, whereas cyclic Axin2 expression is maintained when Notch signaling is impaired (Aulehla et al. 2003; Aulehla and Herrmann 2004), suggesting that WNT acts upstream of Notch in the segmentation clock. However, the exact molecular interplay between the various components of these pathways is not fully understood.

T-box transcription factors as well as FGF and WNT signaling are essential regulators of formation and differentiation or maintenance of paraxial mesoderm in mouse embryos. Mutations in T, Fgfr1, Wnt $3 a$, and Tbx6 cause defects in formation and differentiation of paraxial mesoderm. Loss of $T$ gene function leads to failure of axis development and arrested somite formation (Wilkinson et al. 1990; Herrmann 1995) most likely because of impaired migration of mesodermal cells through the primitive streak (Wilson et al. 1993). The loss of Wnt3a also affects mesodermal cell migration (Yoshikawa et al. 1997). In Wnt3a mutants presumptive paraxial mesoderm cells accumulate beneath the primitive streak and form neural tissues (Yoshikawa et al. 1997). The migratory defect in Wnt $3 a$ mutants might be due to reduced $T$ function because $T$ has been shown to be a direct target of WNT3a signals in the paraxial mesoderm (Yamaguchi et al. 1999). Embryos lacking Tbx6 produce presumptive paraxial mesoderm, but the differentiation of these cells is impaired and supernumerary neural tubes form instead of somites (Chapman and Papaioannou 1998). In embryos lacking Fgfr1 function, presomitic mesoderm formation is impaired, leading to the complete absence of somites and an excess of axial mesoderm (Yamaguchi et al. 1994). In chimeras, Fgfr1 mutant cells accumulate in the primitive streak, fail to colonize the paraxial mesoderm, and differentiate instead to neural tissue (Ciruna et al. 1997). Together these results suggest that WNT and FGF signaling in concert with T-box transcription factors regulate the migration of mesodermal cells and the choice between neural and paraxial mesodermal fates.

WNT signaling as a component of both the segmentation clock and the regulatory network governing paraxial mesoderm differentiation suggests that paraxial meso- 
derm formation and early patterning are tightly coupled. Further support for this idea comes from our analysis of the mouse rib-vertebrae $(r v)$ mutation, which represents a hypomorphic $T b x 6$ allele causing reduced $T b x 6$ expression (Beckers et al. 2000b; Watabe-Rudolph et al. 2002). Reduction of Tbx6 mRNA leads to altered abundance and distribution of mRNAs encoding the Notch pathway components Dll1, Dll3, Lfng, and Notch1 in the presomitic mesoderm, and to somite defects similar to mutations in Notch pathway components (Beckers et al. 2000b). Notably, Dl11, which encodes a critical ligand for Notch in the psm, is severely down-regulated, and $r v$ and a null allele of the Notch ligand Deltal show nonallelic noncomplementation such that double heterozygotes have a mild "rv-phenotype" with complete penetrance (Beckers et al. 2000b). This raises the possibility that Tbx6 not only controls the differentiation of paraxial mesoderm, but might also directly activate expression of Dl11 (and other genes essential for somite patterning) and thereby regulate patterning in the psm.

Here, we show that both T-box- and LEF/TCF-binding sites are essential for activity of the Dl11 promoter in the tailbud and presomitic mesoderm, suggesting that T/TBX6 and WNT signaling directly and synergistically regulate Dll1 transcription in the tailbud and presomitic mesoderm. Thus, WNT signals not only regulate mesoderm formation upstream of $T$ and $T b \times 6$, but also regulate patterning in the psm cooperatively with transcription factors that are themselves targets of WNT activity.

\section{Results and Discussion}

During our analysis of the mouse $r v$ mutation we noticed that Dll1 expression is significantly reduced in the presomitic mesoderm of $r V$ mutant embryos (Beckers et al. 2000b). $r v$ is a hypomorphic allele of the Tbx6 gene (Watabe-Rudolph et al. 2002), which encodes a T-box transcription factor specifically expressed in the primitive streak/tailbud and presomitic mesoderm /Chapman et al. 1996). To address how the complete loss of Tbx6 function affects Dll1, we analyzed Dll1 expression in embryos carrying a targeted null allele of Tbx6. Embryos lacking Tbx6 function have five to seven irregular somites in the prospective hindbrain region, but more posteriorly they form ectopic neural tubes instead of paraxial mesoderm (Chapman and Papaioannou 1998). Dll1 expression in Tbx6-null mutant embryos was severely down-regulated on E8 (Fig. 1b), and Dll1 transcripts were not detected on E8.5 (Fig. 1d) prior to the overt loss of paraxial mesoderm. This suggested that $T b \times 6$ is required to maintain Dll1 expression in the presomitic mesoderm. Brachyury $(T)$ is expressed in the primitive streak and nascent mesoderm, overlapping with Tbx6. $T$ is functionally upstream of $T b \times 6$, since it is required for mesoderm formation (Herrmann et al. 1990), while Tbx6 acts in the maintenance and/or differentiation of paraxial mesoderm (Chapman and Papaioannou 1998). Similar to Tbx6 mutants, E8-E8.5 embryos lacking T showed severely reduced or no Dll1 expression in the psm (Fig. 1f,h). These data raised the question whether Tbx 6 expression depends on $T$. Indeed, we found that Tbx6 is severely down-regulated in embryos lacking $T$ (Fig. 1j). The presence of T sites in introns 1 and 5 of Tbx6 (data not shown) supports the supposition that Tbx6 is a direct target of $T$. The combined expression

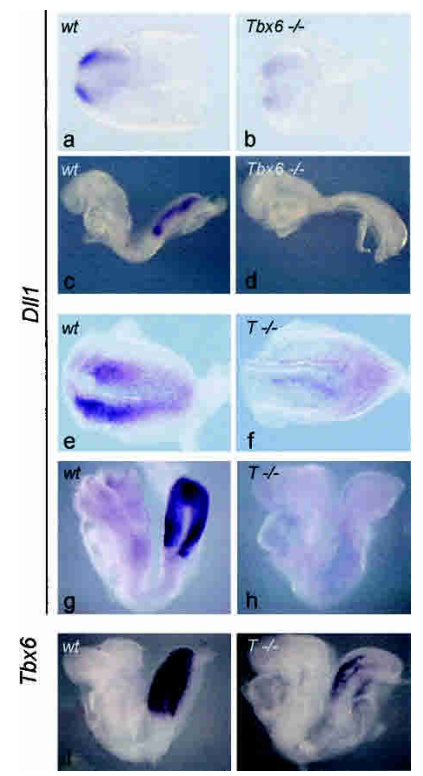

Figure 1. Expression of Dll1 or Tbx6 in $T$ and Tbx6 mutant embryos. In situ hybridization of E8 $(a, b, e, f)$ and E8.5 $(c, d, g-i)$ wild-type $(a, c, e, g, i)$ and homozygous $T(f, h, i)$ and $\operatorname{Tbx} 6(b, d)$ mutant embryos with Dll1 $(a-h)$ and Tbx6 $(i, j)$ probes. Dll1 expression was significantly down-regulated in E8 and not detected in E8.5 T and Tbx6 mutant embryos. In E8.5 T mutant embryos Tbx6 expression was significantly reduced.

data suggest a cascade of factors involved in the control of Dll1 expression.

The reduction of Dll1 transcripts in $T$ mutant embryos, and their reduction and subsequent loss in Tbx6 mutant embryos could indicate that Dl11 is a direct target of these transcriptional regulators. To explore this possibility further, we analyzed the sequence of the promoter region of Dll1 that was previously shown to contain sequences sufficient to drive heterologous gene expression in the psm for potential T-box-binding sites. In this genomic region, which comprises $4.3 \mathrm{~kb}$ upstream of the translational start site, nine putative T-binding sites were identified (Fig. 2A,B). Six of these sites (T2-T7) are located within a 1.4-kb "msd" fragment, which drives heterologous gene expression robustly throughout the psm and in newly formed somites (Beckers et al. 2000a). One $\mathrm{T}$ site (T1) is present at the distal end of the $4.3-\mathrm{kb}$ fragment; two other sites, T8 and T9, are in the proximal region in exon 1 close to the ATG. In addition to the potential T-binding sites, we found eight LEF/TCF-binding sites in the 4.3-kb Dll1 promoter region, four of which are located in the msd fragment (Fig. 2A,B), raising the possibility that WNT activity in addition to T/TBX6 controls expression of Dll1 in the tailbud and psm.

To address whether T, TBX6, and LEF/TCF proteins can activate transcription from the msd promoter fragment, we generated a reporter construct, which expresses luciferase fused to the Dll1 minimal promoter (Beckers et al. 2000a) under the control of msd (msdwtluc), cotransfected this construct together with expression vectors containing cDNAs encoding T, TBX6, or TCF1E into COS7 cells, and determined luciferase activity. Full-length T and TBX6 resulted only in very weak transactivation (data not shown), most likely because of a requirement for unknown cofactors. Therefore, we generated fusions between the DNA-binding do- 


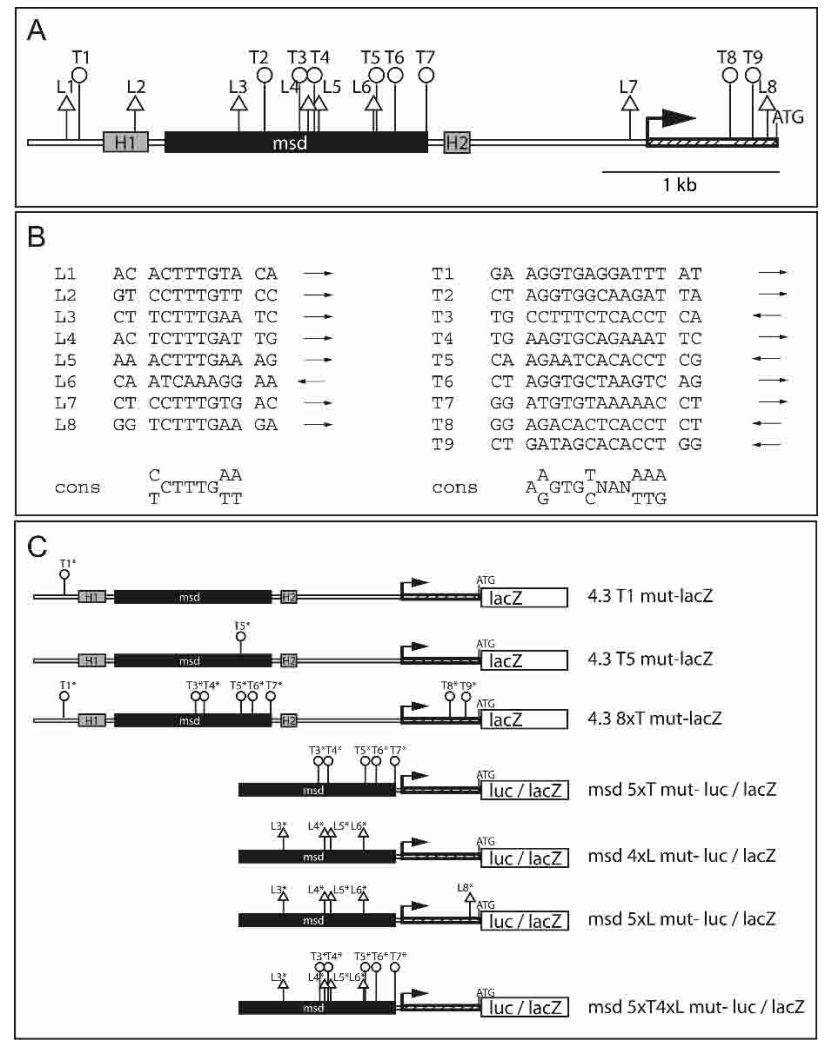

Figure 2. T-box and LEF/TCF sites in the Dll1 promoter and reporter constructs. (A) Schematic representation of the Dll1 promoter region extending $4.3 \mathrm{~kb}$ upstream of the ATG. The position of potential LEF/TCF sites (triangles) and T sites (circles) is indicated. H1 and $\mathrm{H} 2$ refer to the regions of homology detected between the zebrafish deltaD and mouse Dll1 promoters, which direct gene expression into the central nervous system (Beckers et al. 2000a). msd indicates the region directing gene expression in the paraxial mesoderm (Beckers et al. 2000a). (B) Sequences of the potential $\mathrm{T}$ and $\mathrm{LEF} / \mathrm{TCF}$ sites in the Dll1 4.3-kb promoter region. Arrows indicate the orientation of the sites, and the consensus binding sites (cons) for LEF/TCF (Giese et al. 1992; van de Wetering and Clevers 1992) and T-box (Kispert et al. 1995; Kusch et al. 2002) factors are shown below. $(C)$ Schematic representation of mutant reporter gene constructs used in this study. The mutated sites are marked and indicated above the constructs.

mains of these proteins and the VP16 transactivator domain (TVP16 and TBX6VP16, respectively) for further analyses. Either transcription factor alone or in combination with $\beta$-catenin stimulated luciferase activity twoto threefold (Fig. 3A). Coexpression of TVP16 and TCF1E did not enhance luciferase activity above these levels. However, coexpression of TBX6VP16 and TCF1E stimulated a ninefold increase of luciferase activity (Fig. 3A), suggesting that TBX6 and TCF1E cooperate to activate transcription from msd. Coexpression of exogenous $\beta$-catenin with TBX6 and TCF1E did not further stimulate luciferase activity, which is most likely because of endogenous $\beta$-catenin present in COS7 cells (data not shown). To demonstrate that the synergistic effect of TBX6VP16 and LEF/TCF factors is $\beta$-catenin dependent, we cotransfected COS7 cells with TBX6VP16, $\beta$-catenin, and LEF1 or a truncated version of LEF1 (LEF1 $14-89$ ) that is defective for binding of $\beta$-catenin and acts as a dominant-negative protein (Korinek et al. 1997; Hovanes et al. 2001), respectively. In the presence of LEF1 $\Delta 7-89$ no synergistic activation of luciferase activity was observed in contrast to cotransfections of TBX6VP16 and LEF1 either with or without $\beta$-catenin (Fig. 3B). Thus, synergism between TBX6VP16 and LEF/TCF appears to depend on $\beta$-catenin, suggesting that in the presomitic mesoderm Dl11 is directly controlled byTBX6 and WNT signaling.

To demonstrate that the T and LEF/TCF sites mediate transactivation from msd, T sites or LEF/TCF sites or a combination of these sites in msdwtluc were mutated, and mutated constructs were analyzed for luciferase expression. Mutation of all $\mathrm{T}$ sites except for the weakly conserved site T2 (msd5xTmut-luc, Fig. 2A,B) abolished the synergistic activation of luciferase by TBX6VP16 and TCF1E (Fig. 3C). In contrast, mutation of all four LEF/ TCF sites in msd (msd4xLmut-luc) did not affect the cooperative stimulation of transcription by TBX6VP16 and TCF1E (Fig. 3C), suggesting that the LEF/TCF site in the Dll1 minimal promoter fragment close to the ATG (L8, Fig. 2A) might be sufficient to compensate in this assay for the loss of LEF/TCF sites in msd. Indeed, no synergistic activation of luciferase expression was obtained from a construct (msd5xLmut-luc) in which this site was mutated in addition (Fig. 3C). Mutation of five $T$ sites and the four LEF/TCF sites in msd (msd5xT4xLmut-luc) also abolished the synergistic activation. The loss of cooperative transactivation from msd5xTmut-luc, which still contains two potential $\mathrm{T}$ sites, T8 and T9, close to the ATG (Fig. 2A) suggests that these sites do not contribute to activation by T-box transcription factors under these conditions.

To address the significance of the potential $\mathrm{T}$ and LEF/
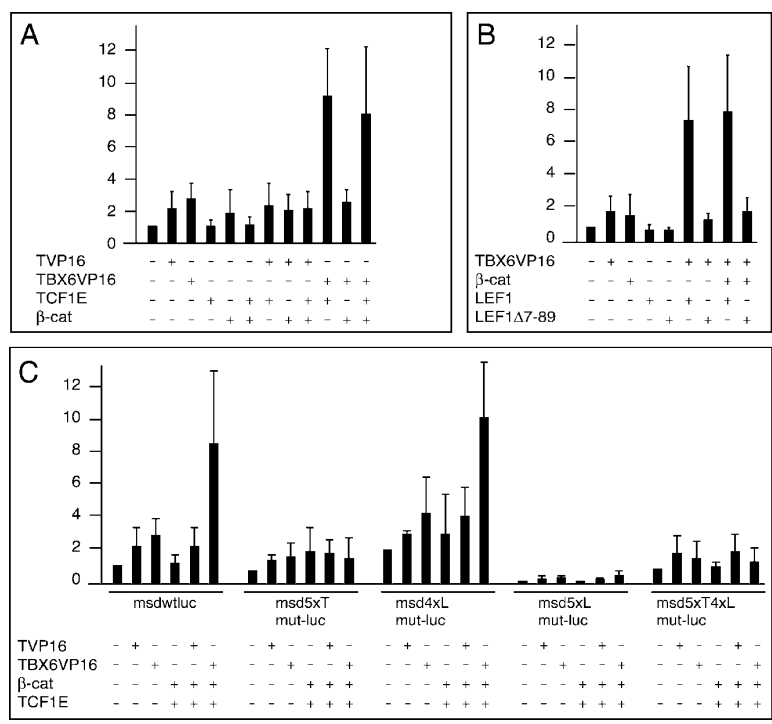

Figure 3. Synergistic activation of transcription from the msd promoter fragment by TBX6 and LEF/TCF. Relative luciferase activity in COS7 cells transfected with reporter gene plasmids and combinations of expression vectors as indicated. Luciferase activity was normalized to activity obtained with the wild-type msd reporter plasmid alone. (A) TBX6VP16 and TCF1E activate the msd promoter synergistically. $(B)$ Synergism between TBX6VP16 and LEF1 depends on the $\beta$-catenin-binding domain of LEF1. $(C)$ Both $\mathrm{T}$ and $\mathrm{LEF} / \mathrm{TCF}$ sites are critical for synergistic activation. For comparison of luciferase activity between different reporter constructs, activity of the mutant promoter constructs was related to the activity obtained with the msd wild-type construct, whose activity was set to one. 


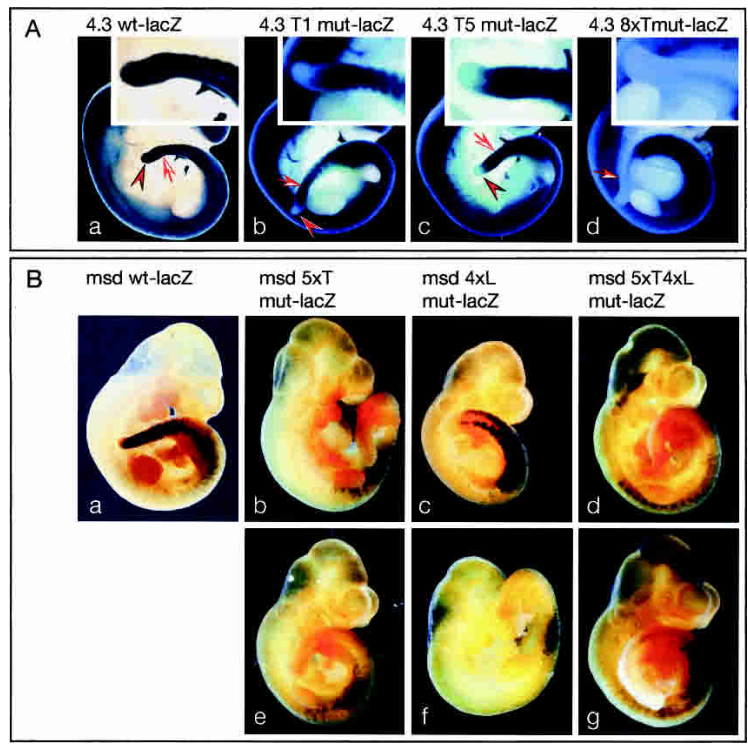

Figure 4. Reporter gene expression in transgenic embryos. Representative examples of $\beta$-galactosidase-stained transgenic embryos obtained after DNA microinjection. (A) Staining patterns in embryos carrying the wild-type (panel $a$ ) and mutant (panels $b-d$ ) $4.3-\mathrm{kb}$ promoter constructs. Note that mutations in either T1 (panel $b$ ) or T5 (panel $c$ ) abolished $\beta$-galactosidase expression specifically in the tailbud (arrowheads). Arrows point to the most recently formed somites. Insets show higher-magnification pictures of the tails. $(B)$ Staining patterns in embryos carrying the wild-type (panel $a$ ) and mutant (panels $b-g$ ) msd promoter constructs. Mutation of either T sites (panels $b, e$ ), or LEF/TCF sites (panels $c$,f), or both (panels $d, g$ ) completely abolished $\beta$-galactosidase in the presomitic mesoderm, which expresses high levels of $\beta$-galactosidase in wild-type msdlacZ transgenic embryos.

TCF sites for Dll1 expression in the presomitic mesoderm of mouse embryos, we generated various lac $Z$ reporter constructs with the $4.3-\mathrm{kb}$ promoter fragment that carried point mutations in individual or multiple $\mathrm{T}$ sites, as well as with the msd promoter carrying mutations in the $\mathrm{T}$ and LEF/TCF sites as before (Fig. 2C), and analyzed lacZ expression in embryos after DNA microinjection into zygotes (Fig. 4). The 4.3-kb promoter fragment recapitulates many aspects of early D111 expression and gives rise to $1 a c Z$ expression in the tailbud, presomitic mesoderm, and somites as well as in the central nervous system (Beckers et al. 2000a). We have previously shown that deletion of the most distal region of the 4.3-kb promoter resulted in loss of transgene expression in the tailbud (Fig. 7 in Beckers et al. 2000a). This region contains the $T$ site $T 1$. When $T 1$ was mutated (4.3T1mut-lacZ, Fig. 2C), lacZ expression in the tailbud was abolished $(n=8)$ (Fig. 4A). Similarly, when T5, which resides in msd and is very similar to the consensus T-binding site (4.3T5mut-lacZ, Fig. 2A,B), was mutated, no lac $Z$ expression in the tailbud was detected $(n=21)$ but psm expression was maintained, suggesting that each of these $\mathrm{T}$ sites is essential for Dll1 expression specifically in the tailbud. Mutation of eight of the nine $\mathrm{T}$ sites in the promoter, excluding the weakly conserved site T2 (4.3 8xTmutlacZ, Fig. 2C), resulted in the complete loss of expression in the tailbud, psm, and newly formed somites $(n=5)$ (Fig. 4A, panel d). The msd fragment drives heterologous gene expression robustly throughout the psm and in newly formed somites (Beck- ers et al. 2000a). When five of the six $\mathrm{T}$ sites in msd were mutated (T3-T7; msd5xTmut-lacZ, Fig. 2C), highly variable $1 a c Z$ expression in the somites but no expression in the psm was detected $(n=7)$ (Fig. 4B, panels b,e). Collectively, these results demonstrate that $\mathrm{T}$ sites in the Dll1 promoter are critical for D111 expression in the tailbud and presomitic mesoderm, strongly supporting the idea that T and/or TBX6 are direct regulators of Dll1 in vivo. Similar to msd with mutated $\mathrm{T}$ sites, mutations in all four LEF/TCF sites in the msd promoter (msd4xLmutlacZ, Fig. 2C) abolished lacZ expression in the psm of transgenic embryos but retained variable somitic expression $(n=11)$ (Fig. 4B, panels $\mathrm{c}, \mathrm{f})$. The combination of mutated $\mathrm{T}$ and LEF/TCF sites (msd 5xT4xLmut-lacZ, Fig. 2C) gave similar results with additional variable ectopic lac $Z$ expression in parts of the central nervous system $(n=10)$ (Fig. 4B, panels d,g). Variable ectopic expression and apparent augmented somitic expression most likely reflect position effects. In addition, loss of LEF/TCF mediated repression in the absence of WNT activity (Roose et al. 1998) might contribute to ectopic or augmented expression from transgene constructs with mutated LEF sites. Consistent with the results of our in vitro studies, these analyses indicate that binding sites both for T-box and LEF/TCF transcription factors and thus binding of both factors are simultaneously required in the msd enhancer to direct transcription in the psm. This strongly suggests that T-box transcription factors and WNT activity cooperatively regulate D111 expression in vivo in the tailbud and psm. Consistent with this interpretation, a targeted mutation of Lef1, which interferes with $\beta$-catenin-dependent activation by LEF/TCF proteins (Galceran et al. 2000), causes down-regulation of Dll1 in the psm, and somite patterning defects (Galceran et al. 2004).

Synergistic activation of the msd promoter in COS cells by TBX6VP16 and LEF/TCF but not by TVP16 and LEF/TCF (Fig. 3A) could indicate that also in vivo TBX6 rather than T directs $D 111$ expression. Thus, the reduction or loss of $D 111$ expression in $T^{-/-}$embryos (Fig. 1f,h) could be an indirect effect of the severe down-regulation of Tbx6 in these mutants (Fig. 1j). Alternatively, T might specifically interact with site $\mathrm{T} 1$, either alone or in combination with (an)other more proximal T site(s), and thus might contribute to expression of Dll1 in the tailbud.

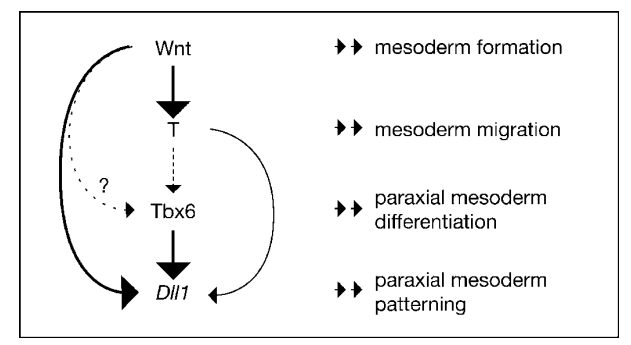

Figure 5. Proposed regulatory network directing Dll1 expression in the tailbud and presomitic mesoderm. WNT signals induce mesoderm formation and Brachyury expression, whose function is essential for migration of mesodermal cells through the primitive streak. $T$ acts upstream of $T b \times 6$, potentially as direct activator, and $T b \times 6$ is required to maintain paraxial mesoderm. Both $\mathrm{T}$ and Tbx6 regulate Dll1 in the tailbud and in the presomitic mesoderm, respectively. WNT signals acting through the canonical Wnt pathway control Dll1 expression in the paraxial mesoderm synergistically with TBX6. (Bold arrows) Proven direct regulation; (dashed arrows) genetically upstream. 
Indeed, recombinant $\mathrm{T}$ protein can bind weakly to $\mathrm{T} 1$ but effectively to a synthetic DNA fragment containing $\mathrm{T} 1$ and the highly conserved site T5 (data not shown), supporting this possibility.

Canonical WNT signals control mesoderm formation and T expression (Takada et al. 1994; Liu et al. 1999; Yamaguchi et al. 1999; Arnold et al. 2000). Our data suggest that WNT activity, in addition to regulating genes required for mesoderm formation, directly controls the expression of genes that are critical for patterning of the paraxial mesoderm (Fig. 5), and further support our previous suggestion that WNT signaling controls the segmentation process (Aulehla et al. 2003). By regulating Dll1 expression, WNT indirectly controls the signaling activity of Notch. Thus, WNT activity regulates multiple aspects of mesoderm development not only by acting high up in the genetic hierarchy that governs mesoderm formation but also by directly cooperating with direct or indirect targets of its own activity.

\section{Materials and methods}

In situ hybridization

Whole mount in situ hybridizations were done by standard procedures.

Mice

Mutant embryos of the genotype $T^{-/-}$and $\mathrm{Tb}_{\mathrm{b}} \mathrm{6}^{-/-}$were derived from intercrosses of the strains BTBR/TF-Ttf/+tf and $T b x b^{t m 1 P a}$, respectively.

Expression and reporter constructs

The reporter genes Dll1tg4.3/lacZ and Dll1' $\mathrm{msd}^{\prime} / \mathrm{lacZ}$ were described previously (Beckers et al. 2000a). Luciferase reporter constructs were generated by replacing $1 a c Z$ with the luciferase gene and the SV40 polyadenylation signal of pGL2 (Promega Corp.). Binding sites for T/TBX6 and TCF/LEF (Fig. 2) were altered using PCR-based site-directed mutagenesis. To disrupt the T/TBX6-binding sites, T1 (position -3973/-3962) was replaced by (AGATAAGGATTT), T2 $(-2278 /-2267)$ by (AGAATCA TATCT), T4 (-2718/-2707) by (CCTTTCTTATCT), T5 (-2634/-2623) by (AAATACAGAAAT), T6 (-2169/-2158) by (AGATACTAAGTC), T7 $(-1997 /-1986)$ by (ATATATAAAAAC), T8 $(-267 /-256)$ by (AGACACTT ATCT), and T9 $(-135 /-124)$ by (GATAGCATATCT). To disrupt the TCF/LEF-binding sites, L3 (-3062/-3056) was replaced by (CTCTAGA), L4 (-2668/-2662) by (CTCAGCT), L5 (-2605/-2599) by (CTCTAGA), L6 $(-2299 /-2293)$ by (TGCACAG), and L7 (-57/-51) by (CTCTAGA). The Tbx6 cDNA was cloned by RT-PCR from RNA of DMSO-treated P19 EC cells. The cloned Tbx6 ORF (1311 bp, GenBank accession no. AY654733) is homologous to the larger ORF of human Tbx6 and was cloned in the EcoRV/HindIII sites of pcDNA3. TBX6VP16 was generated by cloning the VP16 transcriptional activation domain in the BamHI/NotI site of pcDNA3/Tbx6 deleting the last four amino acids of the Tbx6 ORF. TVP16 was made by cloning the T-domain (amino acids 1-229) into the EcoRI/BamHI sites of pSG5 (Stratagene) and adding a PCR fragment containing a nuclear localization signal followed by the VP16 transcriptional activation domain cloned in frame using the BamHI/NotI sites of pSG5. Constructs pCS2+/TCF1E, pCS2+/LEF1, pCS2+/LEF1 $\Delta \mathrm{N}$ and $\mathrm{pCS} 2+/ \beta-$ cateninS33A were provided by A. Hecht (University of Freiburg, Freiburg, Germany).

Cell culture, transient transfection, and reporter gene assays

African green monkey (COS7) cells (ATCC number CRL-1651) were grown in DMEM (Sigma) supplemented with $10 \%$ FBS (Invitrogen) and $1 \times$ penicillin-streptomycin-glutamine (Invitrogen) at $37^{\circ} \mathrm{C}$ and $5 \% \mathrm{CO}_{2}$. Cells were seeded at a density of $4 \times 10^{5}$ cells/well in six-well plates 20 $\mathrm{h}$ before transfection. Cells were cotransfected with $1 \mu \mathrm{g}$ of DNA of expression and reporter plasmids. Total amounts of DNA were kept constant by adding empty expression vector where needed. pCMV- $\beta$-galactosidase was cotransfected as an internal control to normalize for differences in transfection efficiency. Transfections were performed with Lipofectamine 2000 reagent (Invitrogen) according to the manufacturer's recommendations, and cells were harvested after $24 \mathrm{~h}$. Cell were lysed in $400 \mu \mathrm{L}$ of $50 \mathrm{mM}$ Tris phosphate (pH 7.8), $250 \mathrm{mM} \mathrm{KCl}, 0.1 \%$ Nonidet
P-40, and $10 \%$ glycerol on ice for 20 min. Firefly luciferase and $\beta$-galactosidase activities were measured in a Luminoskan Ascent luminometer (Thermo Labsystems). The reporter gene activities shown are average values, obtained from at least four independent experiments.

DNA microinjection and $\beta$-galactosidase staining

Transgenic embryos were produced by pronuclear injections of linearized DNA constructs into fertilized eggs derived from strain $\mathrm{FVB} / \mathrm{N}$ according to standard techniques, dissected at the indicated stages, and processed for $\beta$-galactosidase staining as described (Beckers et al. 2000a).

\section{Acknowledgments}

We thank Manuela Scholze for excellent technical assistance, A. Hecht and R. Kemler for reagents, V. Papaioannou for Tbx6 mutant mice, and J. Galceran and R. Grosschedl for communicating results prior to publication. This work was supported by grants of the Deutsche Forschungsgemeinschaft to B.G.H. (SFB592) and A.G. (Go449/5-2).

\section{References}

Arnold, S.J., Stappert, J., Bauer, A., Kispert, A., Herrmann, B.G., and Kemler, R. 2000. Brachyury is a target gene of the Wnt $/ \beta$-catenin signaling pathway. Mech. Dev. 91: 249-258.

Aulehla, A. and Herrmann, B.G. 2004. Segmentation in vertebrates: Clock and gradient finally joined. Genes \& Dev. 18: 2060-2067.

Aulehla, A., Wehrle, C., Brand-Saberi, B., Kemler, R., Gossler, A., Kanzler, B., and Herrmann, B.G. 2003. Wnt3a plays a major role in the segmentation clock controlling somitogenesis. Dev. Cell 4: 395-406.

Beckers, J., Caron, A., Hrabe de Angelis, M., Hans, S., Campos-Ortega, J.A., and Gossler, A. 2000a. Distinct regulatory elements direct Deltal expression in the nervous system and paraxial mesoderm of transgenic mice. Mech. Dev. 95: 23-34.

Beckers, J., Schlautmann, N., and Gossler, A. 2000b. The mouse ribvertebrae mutation disrupts anterior-posterior somite patterning and genetically interacts with a Deltal null allele. Mech. Dev. 95: 35-46.

Chapman, D.L. and Papaioannou, V.E. 1998. Three neural tubes in mouse embryos with mutations in the T-box gene Tbx6. Nature 391: 695697.

Chapman, D.L., Agulnik, I., Hancock, S., Silver, L.M., and Papaioannou, V.E. 1996. Tbx6, a mouse T-Box gene implicated in paraxial mesoderm formation at gastrulation. Dev. Biol. 180: 534-542.

Ciruna, B.G., Schwartz, L., Harpal, K., Yamaguchi, T.P., and Rossant, J. 1997. Chimeric analysis of fibroblast growth factor receptor-1 (Fgfr1) function: A role for FGFR1 in morphogenetic movement through the primitive streak. Development 124: 2829-2841.

Conlon, R.A., Reaume, A.G., and Rossant, J. 1995. Notch1 is required for the coordinate segmentation of somites. Development 121: 15331545.

Dale, J.K., Maroto, M., Dequeant, M.L., Malapert, P., McGrew, M., and Pourquie, O. 2003. Periodic Notch inhibition by Lunatic fringe underlies the chick segmentation clock. Nature 421: 275-278.

del Barco Barrantes, I., Elia, A.J., Wünsch, K., Hrabe De Angelis, M., Mak, T.W., Rossant, R., Conlon, R.A., Gossler, A., and de la Pompa, J.-L. 1999. Interaction between L-fringe and Notch signaling in the regulation of boundary formation and posterior identity in the presomitic mesoderm of the mouse. Curr. Biol. 9: 470-480.

Dunwoodie, S.L., Clements, M., Sparrow, D.B., Sa, X., Conlon, R.A., and Beddington, R.S. 2002. Axial skeletal defects caused by mutation in the spondylocostal dysplasia/pudgy gene Dll3 are associated with disruption of the segmentation clock within the presomitic mesoderm. Development 129: 1795-1806.

Forsberg, H., Crozet, F., and Brown, N.A. 1998. Waves of mouse Lunatic fringe expression, in four-hour cycles at two-hour intervals, precede somite boundary formation. Curr. Biol. 8: 1027-1030.

Galceran, J., Miyashita-Lin, E.M., Devaney, E., Rubenstein, J.L., and Grosschedl, R. 2000. Hippocampus development and generation of dentate gyrus granule cells is regulated by LEF1. Development 127: 469-482.

Galceran, J., Sustmann, C., Hsu, S.-C., Folberth, S., and Rudolf Grosschedl, R. 2004. LEF1-mediated regulation of Delta-like1 links Wnt and Notch signaling in somitogenesis. Genes \& Dev. (this issue). 
Giese, K., Cox, J., and Grosschedl, R. 1992. The HMG domain of lymphoid enhancer factor 1 bends DNA and facilitates assembly of functional nucleoprotein structures. Cell 69: 185-195.

Herrmann, B.G. 1995. The mouse Brachyury (T) gene. Sem. Dev. Biol. 6: 385-394.

Herrmann, B.G., Labeit, S., Poustka, A., King, T.R., and Lehrach, H. 1990. Cloning of the $T$ gene required in mesoderm formation in the mouse. Nature 343: 617-622.

Holley, S.A., Geisler, R., and Nusslein-Volhard, C. 2000. Control of her1 expression during zebrafish somitogenesis by a $\delta$-dependent oscillator and an independent wave-front activity. Genes \& Dev. 14: 1678 1690.

Hovanes, K., Li, T.W., Munguia, J.E., Truong, T., Milovanovic, T., Lawrence Marsh, J., Holcombe, R.F., and Waterman, M.L. 2001. $\beta$-Catenin-sensitive isoforms of lymphoid enhancer factor-1 are selectively expressed in colon cancer. Nat. Genet. 28: 53-57.

Hrabe de Angelis, M., McIntyre II, J., and Gossler, A. 1997. Maintenance of somite borders in mice requires the Delta homologue DIIl. Nature 386: 717-721.

Jen, W., Gawantka, V., Pollet, N., Niehrs, C., and Kintner, C. 1999. Periodic repression of notch pathway genes governs the segmentation of Xenopus embryos. Genes \& Dev. 13: 1486-1499.

Jiang, Y.J., Aerne, B.L., Smithers, L., Haddon, C., Ish-Horowicz, D., and Lewis, J. 2000. Notch signaling and the synchronization of the somite segmentation clock. Nature 408: 475-479.

Jouve, C., Palmeirim, I., Henrique, D., Beckers, J., Gossler, A., Ish Horowcz, D., and Pourquié, O. 2000. Notch signaling is required for cyclic expression of the hairy-like gene HES1 in the presomitic mesoderm. Development 127: 1421-1429.

Kispert, A., Koschorz, B., and Herrmann, B.G. 1995. The T protein encoded by Brachyury is a tissue-specific transcription factor. EMBO $\mathrm{T}$. 14: 4763-4772.

Korinek, V., Barker, N., Morin, P.J., van Wichen, D., de Weger, R., Kinzler, K.W., Vogelstein, B., and Clevers, H. 1997. Constitutive transcriptional activation by a $\beta$-catenin-Tcf complex in $\mathrm{APC}^{-/-}$colon carcinoma. Science 275: 1784-1787.

Kusch, T., Storck, T., Walldorf, U., and Reuter, R. 2002. Brachyury proteins regulate target genes through modular binding sites in a cooperative fashion. Genes \& Dev. 16: 518-529.

Liu, P., Wakamiya, M., Shea, M.J., Albrecht, U., Behringer, R.R., and Bradley, A. 1999. Requirement for Wnt3 in vertebrate axis formation. Nat. Genet. 22: 361-365.

Palmeirim, I., Henrique, D., Ish-Horowicz, D., and Pourquie, O. 1997. Avian hairy gene expression identifies a molecular clock linked to vertebrate segmentation and somitogenesis. Cell 91: 639648.

Roose, J., Molenaar, M., Peterson, J., Hurenkamp, J., Brantjes, H., Moerer, P., van de Wetering, M., Destree, O., and Clevers, H. 1998. The Xenopus Wnt effector XTcf-3 interacts with Groucho-related transcriptional repressors. Nature 395: 608-612.

Sawada, A., Fritz, A., Jiang, Y., Yamamoto, A., Yamasu, K., Kuroiwa, A., Saga, Y., and Takeda, H. 2000. Zebrafish Mesp family genes, mesp-a and mesp-b are segmentally expressed in the presomitic mesoderm, and Mesp-b confers the anterior identity to the developing somites. Development 127: 1691-1702.

Serth, K., Schuster-Gossler, K., Cordes, R., and Gossler, A. 2003. Transcriptional oscillation of lunatic fringe is essential for somitogenesis. Genes \& Dev. 17: 912-925.

Takada, S., Stark, K.L., Shea, M.J., Vassileva, G., McMahon, J.A., and McMahon, A.P. 1994. Wnt-3a regulates somite and tailbud formation in the mouse embryo. Genes \& Dev. 8: 174-189.

van de Wetering, M. and Clevers, H. 1992. Sequence-specific interaction of the HMG box proteins TCF-1 and SRY occurs within the minor groove of a Watson-Crick double helix. EMBO J. 11: 30393044 .

Watabe-Rudolph, M., Schlautmann, N., Papaioannou, V.E., and Gossler, A. 2002. The mouse rib-vertebrae mutation is a hypomorphic Tbx6 allele. Mech. Dev. 119: 251-256.

Wilkinson, D.G., Bhatt, S., and Herrmann, B.G. 1990. Expression pattern of the mouse $T$ gene and its role in mesoderm formation. Nature 343: 657-659.

Wilson, V., Rashbass, P., and Beddington, R.S. 1993. Chimeric analysis of $\mathrm{T}$ (Brachyury) gene function. Development 117: 1321-1331.
Yamaguchi, T.P., Harpal, K., Henkemeyer, M., and Rossant, J. 1994. $f g f r-1$ is required for embryonic growth and mesodermal patterning during mouse gastrulation. Genes \& Dev. 8: 3032-3044.

Yamaguchi, T.P., Takada, S., Yoshikawa, Y., Wu, N., and McMahon, A.P. 1999. T (Brachyury) is a direct target of Wnt3a during paraxial mesoderm specification. Genes \& Dev. 13: 3185-3190.

Yoshikawa, Y., Fujimori, T., McMahon, A.P., and Takada, S. 1997. Evidence that absence of Wnt-3a signaling promotes neuralization instead of paraxial mesoderm development in the mouse. Dev. Biol. 183: 234-242. 


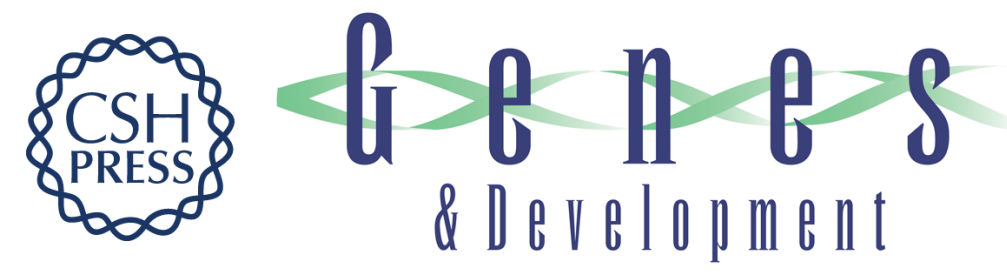

\section{WNT signaling, in synergy with T/TBX6, controls Notch signaling by regulating DIl1 expression in the presomitic mesoderm of mouse embryos}

Michael Hofmann, Karin Schuster-Gossler, Masami Watabe-Rudolph, et al.

Genes Dev. 2004, 18:

Access the most recent version at doi:10.1101/gad.1248604

References This article cites 38 articles, 16 of which can be accessed free at: http://genesdev.cshlp.org/content/18/22/2712.full.html\#ref-list-1

License

Email Alerting Service

Receive free email alerts when new articles cite this article - sign up in the box at the top right corner of the article or click here.

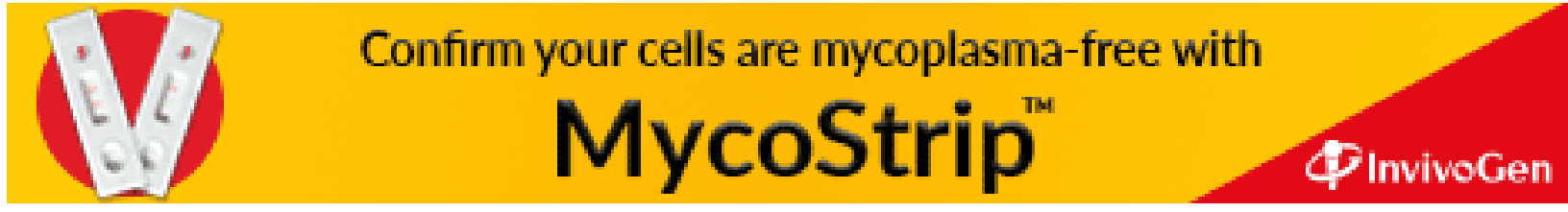

